\title{
Lensing-Induced Structure of Submillimeter Sources: Implications for the Microwave Background
}

\author{
Evan Scannapieco, Joseph Silk, and Jonathan C. Tan \\ Departments of Physics and Astronomy, University of California, Berkeley, CA 94720-7304
}

\begin{abstract}
We consider the effect of lensing by galaxy clusters on the angular distribution of submillimeter wavelength objects. While lensing does not change the total flux and number counts of submillimeter sources, it can affect the number counts and fluxes of flux-limited samples. Therefore imposing a flux cut on point sources not only reduces the overall Poisson noise, but imprints the correlations between lensing clusters on the unresolved flux distribution. Using a simple model, we quantify the lensing anisotropy induced in flux-limited samples and compare this to Poisson noise. We find that while the level of induced anisotropies on the scale of the cluster angular correlation length is comparable to Poisson noise for a slowly evolving cluster model, it is negligible for more realistic models of cluster evolution. Thus the removal of point sources is not expected to induce measurable structure in the microwave or far-infrared backgrounds.
\end{abstract}

Subject headings: gravitational lensing - galaxies: clusters: general - infrared: galaxies - cosmic microwave background 


\section{Introduction}

Due to the steep slope of the Rayleigh-Jeans portion of their emission spectra, dusty galaxies have submillimeter luminosities that are greatly enhanced as a function of redshift. This results in large negative submillimeter K-corrections, causing the number of galaxies as a function of observed flux to be roughly constant between redshifts 1 and 10 (Franceschini et al. 1991; Blain \& Longair 1993). Thus observations that can resolve submillimeter sources at $z=1$ should be able to detect the full history of these objects, and one would expect submillimeter source counts to have a much steeper slope as a function of limiting flux than source counts in other wavebands.

While this strong dependence on limiting flux had long been predicted (Blain \& Longair 1993; Blain \& Longair 1996), the overall surface density of these objects remained uncertain to within a factor of a thousand until recently. Using the the new Submillimeter Common User Bolometer Array (SCUBA) (Holland et al. 1999) on the James Clerk Maxwell Telescope, several groups (Barger et al. 1998; Eales et al. 1993; Hughes et al. 1998; Smail, Ivison, \& Blain 1997) have detected objects at $850 \mu \mathrm{m}$ with fluxes of a few mJy. Meanwhile, parallel investigations utilising the FIRAS instrument on COBE (Puget et al. 1996; Fixsen et al. 1998; Hauser et al. 1998), DIRBE combined with IRAS (Schlegel, Finkbeiner and Davis 1998) and TeV gamma ray constraints via pair production against the IR background (Biller et al. 1998) have begun mapping out the extra galactic background from $\sim 5$ to $1000 \mu \mathrm{m}$ wavelengths. Thus the past few years have not only given us our first look at the high-luminosity submillimeter universe, but allowed us to place strong constraints on the unresolved distribution as well. This has allowed several authors to investigate the effects of confusion noise from unresolved sources both on galaxy surveys and measurements of the cosmic microwave background (CMB) (Blain, Ivison, \& Smail 1998; Blain et al. 1998; Scott \& White 1998).

The steepness of submillimeter source counts also makes them highly sensitive to the effects of gravitational lensing. As a gravitational lens amplifies the flux of objects behind it while at the same time reducing the effective volume that is being seen, only objects with sufficiently steep source counts display a statistical increase in bright-end counts when lensed. Thus one would expect the lensed fraction of bright submillimeter-wave sources to be much larger that the fraction in the optical and radio wavebands (Blain 1996). This sensitivity has motivated several SCUBA searches for objects behind galaxy clusters, using lensing to boost resolved source counts. It has also led to investigations of the effect of lensing by both galaxies and galaxy clusters on overall number counts and the prospect of measuring the degree of lensing from all-sky microwave experiments (Blain 1996; Blain 1997; Blain 1998). 
In addition to changing the number counts of bright objects, lensing induces structure in the unresolved source distribution. As the clumping of matter cannot affect the total flux or number of sources, lensing skews the flux distribution by making certain sources brighter while at the same time preserving the total flux and number of objects. Thus imposing a flux cut causes a larger flux decrement in the areas behind gravitational lenses. As clusters of galaxies are known to have large angular correlations (Dalton et al. 1984; Tadros, Efstathiou, \& Dalton 1998), the removal of resolved point sources will imprint a correlated decrement on the unresolved distribution of submillimeter sources.

As future experiments such as the Planck satellite (Bersanelli et al. 1996) will be making sensitive all-sky measurements at microwave and submillimeter wavelengths and will use flux-limited samples to investigate the structure of background emission, it is important that this effect be quantified. While many elements contributing to this structure remain uncertain, current cluster and submillimeter observations have improved our knowledge to the point where this quantification is now possible.

In this work we develop a simple model of lensing by clusters, appropriate to the degree at which cluster evolution is understood. We then combine our lensing model with a semi-empirical model of the submillimeter universe (Tan, Silk \& Balland 1999, hereafter TSB), based on recent observations. This leads to a quantification of induced structure by lensing that is useful in determining the extent to which this effect needs to be considered in future observations.

The structure of this work is as follows: In Section 2 we discuss various models of the lensing of high redshift objects by clusters. In Section 3 we examine the number and spectral evolution of the submillimeter sources themselves and apply our lensing models to predict the number counts, fluxes, and clustering properties of lensed sources. In Section 4 we compare these predictions to Poisson noise and discuss implications for background experiments. Conclusions are given in Section 5.

\section{Models of Gravitational Lensing}

\subsection{Lensing by Clusters}

The effect of gravitational lensing on the number densities of distant sources can be described by $F(A, z) d A$, the probability that a source at redshift $z$ will have its flux density amplified by a factor between $A$ and $A+d A$. For any distribution of objects

$$
\int_{0}^{\infty} F(A, z) d A=1=\int_{0}^{\infty} A F(A, z) d A,
$$


the first equality being a statement of conservation of probability, and the second following from flux conservation. Note that while naively one would expect lensing to cause only amplification, the requirement that the mean flux density be unaffected by the clumping of matter in the universe results in a net deamplification of unlensed objects (Weinberg 1976; Dyer \& Roeder 1973). Thus for all redshifts $F(A, z)$ is a skewed distribution with a maximum slightly less than one and a long tail corresponding to strong lensing events.

By integrating over the comoving mass distribution of clusters, $n(M, z), F(A, z)$ can be determined as

$$
F(A, z)=\pi \frac{d}{d A} \int_{0}^{z} d z^{\prime} \int_{0}^{\infty} d M b(A, M)^{2}\left(1+z^{\prime}\right)^{2} \frac{d n\left(M, z^{\prime}\right)}{d M} \frac{d r}{d z^{\prime}}
$$

where $d r$ is a comoving radial distance element, and $b$ is the proper impact parameter for a source at redshift $z$ and a lens mass $M$ located at a redshift $z^{\prime}$ (Peacock 1982).

The proper impact parameter is the angular size distance to the lens times the angle of deflection by that lens, and is dependent on the geometry of the lensing distribution. Fortunately, the most simple assumption, the $\rho \propto r^{-2}$ isothermal sphere distribution, has been shown both theoretically and observationally to be a good fit for intermediate radii between 0.1 and $1.5 h^{-1} \mathrm{Mpc}$, where $h$ is the Hubble constant in units of $100 \mathrm{~km} \mathrm{~s}^{-1} \mathrm{Mpc}^{-1}$ (Tyson \& Fischer 1995; Navarro, Frenck \& White 1996). For large magnifications this model gives (Peacock 1986)

$$
b(A, M)=\frac{8 \pi \sigma_{v}^{2}}{c^{2}} \frac{D\left(0, z^{\prime}\right) D\left(z^{\prime}, z\right)}{D(0, z)} \frac{1}{A},
$$

were $\sigma_{v}$ is the velocity dispersion of the lens, $D\left(0, z^{\prime}\right)$ is the angular size distance to the lens, $D(0, z)$ is the angular size distance to the lensed object, and $D\left(z^{\prime}, z\right)$ is the angular size distance from the lensed object to the lens. For clusters, $\sigma_{v}=860 \mathrm{~m}^{1 / 2} \mathrm{~km} / \mathrm{sec}$, where $m$ is the mass of the cluster within $1.5 \mathrm{Mpc} h^{-1}$ in units of $6 \times 10^{14} M_{\odot} h^{-1}$. Strictly speaking, the angular size distances in Eq. 3 are the Dyer-Roeder distances which account for the inhomogeneity of the lensing objects. For our purposes here, however, we use the homogeneous distances (Young et al. 1980).

Bahcall, Fan, \& Cen (1997) have used the CNOC/EMSS sample of high-redshift clusters to determine the observed evolution of cluster abundances. For massive clusters with $m \geq 1.05$, the evolution in redshift is given by $\log n(z) \propto-z$ with $\log (n(z=0) / n(z=0.5))=0.7 \pm 0.3(1 \sigma)$ (Fan, Bahcall \& Cen 1997, hereafter FBC). Carlberg et al. (1996) give similar results. One could envision modelling the evolution of clusters from a Press-Schechter (Press \& Schechter 1974) viewpoint, counting the number of peaks above a certain threshold as a function of redshift and cosmology. The problem 
with this approach, however, is that while the evolution of the number density is only weakly dependent on the cosmological parameters, it is strongly dependent on the unknown amplitude of mass fluctuations on the $8 h^{-1} \mathrm{Mpc}$ scale, $\sigma_{8}$ (FBC). As $\sigma_{8}$ is uncertain from observations, a simple fit to data is the best we can hope for even if we fix the other cosmological parameters.

For our purposes then, we adopt a simple model for the number and redshift evolution of clusters that retains the Press-Schechter form but is fit to the observed number density and evolution of clusters

$$
\frac{d n}{d m}=n_{0} m^{-7 / 4} e^{-\left(m^{1 / 2}(z \alpha+\beta)\right)} .
$$

Here the $m^{1 / 2}$ dependence in the exponential and overall $m^{-7 / 4}$ dependence corresponds to a mass density power spectrum $P(k) \propto k^{-1.5}$ around $8 h^{-1} \mathrm{Mpc}$, where cold dark matter models give $P(k) \propto k^{-1.4}$ (Bardeen et al. 1986) We fix these parameters to the $n(>M)$ relation for clusters as calculated in Carlberg et al. (1997), which used the Canadian Network for Observational Cosmology (CNOC) (Yee, Ellingson, \& Carlberg 1996) cluster sample drawn from the Extended Medium Sensitivity Survey (EMSS) (Henry et al. 1992) as well as the ESO Cluster Survey (Mazure et al. 1996) and other cluster data sets (Henry \& Arnaud 1991, hereafter HA; Eke, Cole, \& Frenck 1996, hereafter ECF). This comparison yields $\beta=10$ and $n_{0} \times e^{-\beta}=10^{-6} h^{-3} \mathrm{Mpc}^{-3}$. For this set of parameters the $\log (n(z=0) / n(z=0.5))$ comparison of FBC quoted above corresponds to $\alpha=2.9 \pm 1.1(1 \sigma)$. Note, however, that cluster evolution fits have been carried out by other authors and there is some disagreement between results (Blanchard \& Bartlett 1998; Sadat, Blanchard, \& Oukbir 1998). In Fig. 1 we compare the data compiled in Carlberg et al. (1997). with the "best-fit" FBC, $\alpha=2.9$ evolution model and a slowly evolving, $\alpha=0.7$ model that we consider in further detail below.

Modelling clusters in this manner, we find that for large amplifications $F(z, A)=a(z) A^{-3}$ where

$$
a(z)=0.007 \int_{0}^{z} d z^{\prime} \frac{d r}{d z^{\prime}} \frac{S\left(\eta\left(z^{\prime}\right)\right)^{2} S\left(\eta\left(z-z^{\prime}\right)\right)^{2}}{S(\eta(z))^{2}} \int_{m_{\min }}^{m_{\max }} d m m^{1 / 4} e^{10} e^{-\left(m^{1 / 2}(z \alpha+10)\right)},
$$

where $\eta$ is a dimensionless comoving distance $(\eta=r H / c), S(\eta)=\eta$ in a flat universe, and $S(\eta)=\sinh \left(\eta \sqrt{1-\Omega_{\mathrm{M}}-\Omega_{\Lambda}}\right) / \sqrt{1-\Omega_{\mathrm{M}}-\Omega_{\Lambda}}$ in an open universe. Note that this expression is comparable to the model considered by (Pei 1995), which he derived from observations of the empirical distribution of dense groups and rich clusters (Zabludoff et al. 1993), although our approach is quite different. Throughout this paper we fix $M_{\min }=3 \times 10^{14} M_{\odot}$ and $M_{\max }=1 \times 10^{15} M_{\odot}$.

In Fig. 2 2 we plot this function for three different cosmologies in the upper panel, and 


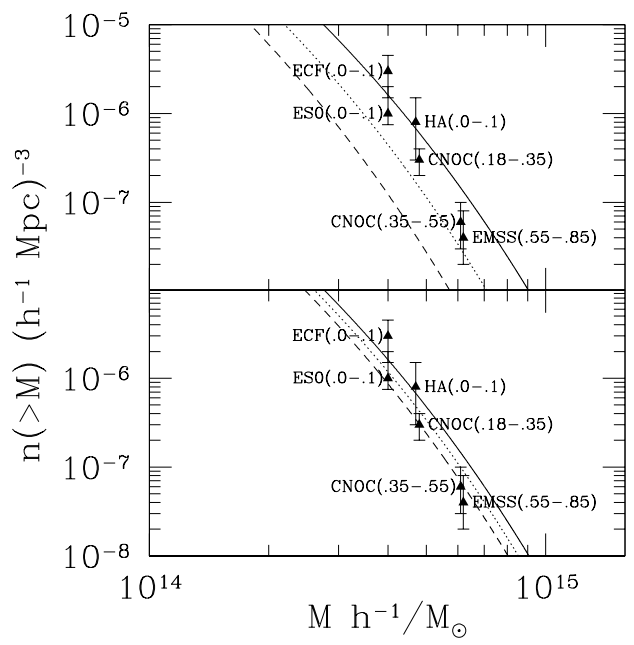

Fig. 1. - The $n(>M)$ relation as calculated fit to the data compiled by Carlberg et al. (1997), with redshift ranges indicated and acronyms are as described in Sec 2.1. The solid lines are $z=0$, the dotted lines are $z=0.5$, and the dashed lines are $z=1.0$. The upper panel shows the FBC $(\alpha=2.9)$ model and the lower panel shows the slowly-evolving $(\alpha=0.7)$ model. $M$ is the mass of the cluster within $1.5 h^{-1} \mathrm{Mpc}$.

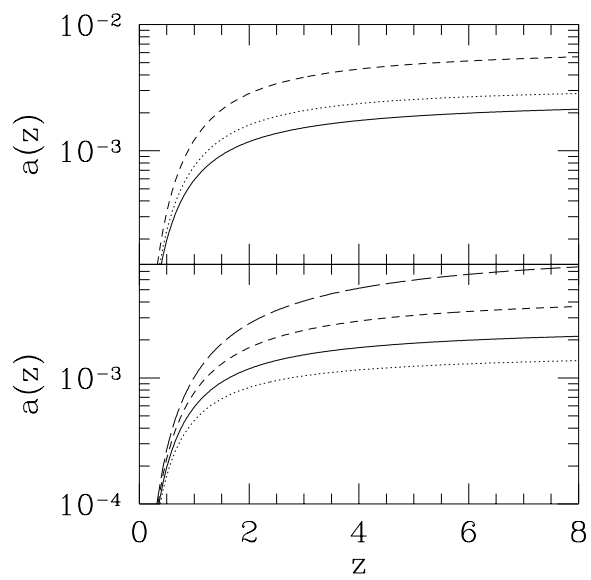

Fig. 2.- Upper panel: Plots of $a(z)$, a measure of the probability of strong lensing as function of redshift, for three different cosmological models: a flat, $\Omega_{\mathrm{M}}=1$ universe (solid), an open model with $\Omega_{\mathrm{M}}=0.3$ (dotted), and a flat cosmological constant model with $\Omega_{\mathrm{M}}=0.3$ (dashed). In all cases $\alpha=2.9$. Lower panel: Plots of $a(z)$ for Einstein-de Sitter cosmologies with four different models for the evolution of clusters: $\alpha=4.0$ (dotted), $\alpha=2.9$ (solid), $\alpha=1.8$ (short-dashed) and $\alpha=0.7$ (long-dashed). 
for four different cluster evolution models in the lower panel. Notice that at moderate redshifts the cosmological constant model gives slightly more than twice the lensing of the Einstein-de Sitter model, while the slow-evolution model of lensing is four times higher than the FBC evolutionary model. Thus while the degree of lensing is quite sensitive to cosmological parameters (Carroll \& Press 1992), lensing by galaxy clusters is even more sensitive to the evolution of the cluster population itself.

\subsection{Clustering of Clusters}

The effect of gravitational lensing on the clustering of a distribution of sources depends on the probability that two sources at redshift $z_{1}$ and $z_{2}$ separated by an angle $\theta$ will be lensed by factors between $A_{1}$ and $A_{1}+d A_{1}$ and $A_{2}$ and $A_{2}+d A_{2}: F\left(A_{1}, z_{1}, A_{2}, z_{2}, \theta\right) d A_{1} d A_{2}$. Similar probability and flux conservation relations to Eq. 11 hold for this distribution:

$$
\int_{0}^{\infty} d A_{1} \int_{0}^{\infty} d A_{2} F\left(A_{1}, z_{1}, A_{2}, z_{2}, \theta\right)=1=\int_{0}^{\infty} A_{1} d A_{1} \int_{0}^{\infty} A_{2} d A_{2} F\left(A_{1}, z_{1}, A_{2}, z_{2}, \theta\right) .
$$

In this case $F\left(A_{1}, z_{1}, A_{2}, z_{2}, \theta\right)$ can be determined as

$$
\begin{aligned}
F\left(A_{1}, z_{1}, A_{2}, z_{2}, \theta\right)=\frac{d}{d A_{1}} \frac{d}{d A_{2}} & \int_{0}^{z_{1}} \frac{d r}{d z_{1}^{\prime}} d z_{1}^{\prime}\left(1+z_{1}^{\prime}\right)^{2} \times \\
& \int_{0}^{z_{2}} \frac{d r}{d z_{2}^{\prime}} d z_{2}^{\prime}\left(1+z_{1}^{\prime}\right)^{2} \pi b\left(A_{1}\right)^{2} \pi b\left(A_{2}\right)^{2} n^{2}\left(z_{1}, z_{2}, \theta\right),
\end{aligned}
$$

where $n^{2}\left(z_{1}, z_{2}, \theta\right)$ is the product of the number density of clusters at redshifts $z_{1}$ and $z_{2}$ separated by an angle $\theta$.

Strictly speaking

$$
n^{2}\left(z_{1}, z_{2}, \theta\right)=n\left(z_{1}\right) n\left(z_{2}\right)\left[1+\xi\left(r\left(z_{1}, z_{2}, \theta\right)\right)\right]
$$

where $\xi(r)$ is the cluster spatial correlation function, and $r\left(z_{1}, z_{2}, \theta\right)$ is the comoving distance between two clusters as a function of their redshifts and separation. There are however a number of difficulties in implementing such an approach, as not only is $r$ dependent on unknown cosmological parameters, but the spatial correlation function of clusters is subject to a number of observational uncertainties. While several authors have fit the optical cluster-cluster correlations with a power law of the form $\eta=\left(r / r_{0}\right)^{-\gamma}$ with $-2.2 \leq \gamma \leq-1.8$ (Peacock \& West 1992; Dalton et al. 1994), the cluster-cluster correlation length $r_{0}$ has remained controversial. Here values are much more uncertain, ranging from $15 h^{-1} \mathrm{Mpc}$ (Nichol et al. 1992; Dalton et al. 1994) to $25 h^{-1}$ Mpc (Peacock \& West 1992; Abadi, Lambas, \& Muriel 1998), with various dependences on cluster mass and mean intercluster 
separation (Bahcall \& Soneira 1983; Bahcall \& Cen 1992). To compound this problem, the clustering of clusters should be more severe at higher redshifts due to the fact that the first density peaks to collapse were more rare than those collapsing recently.

Due to these uncertainties, we avoid implementing the spatial correlation function and approximate the number density of objects as

$$
n^{2}\left(z_{1}, z_{2}, \theta\right) \approx n\left(z_{1}\right) n\left(z_{2}\right)[1+w(\theta)]
$$

with $w(\theta)=\left(\theta / \theta_{0}\right)^{1+\gamma}$, with $\theta_{0}=1.1^{\circ}$ observed in the APM survey (Dalton et al. 1994). Note however, smaller values of $\theta_{0}$ may be more appropriate for lensing due to the larger redshift range at which clusters have an observable effect. Thus our choice for the angular correlation function of lensing clusters should be considered an upper limit.

As the form of the peak of $F(A, z)$ at amplifications slightly less than unity has little effect on the results of lensing, Blain (1996), following Vietri \& Ostriker (1982), examined a simple model for $F$ :

$$
F=H(z) \delta\left(A-A_{0}(z)\right)+A^{-3} a(z) \theta\left(A-A_{\min }\right) \theta\left(A_{\max }-A\right)
$$

where $H(z)$ and $A(z)$ are fixed by the conservation relations Eq. 1, and $\theta$ is the Heaviside step function, which is used to exclude low magnifications at which we would expect Eq. 2 to break down and high magnifications at which the isothermal sphere approximation is invalid. Working to first order in $a(z)$, this gives

$$
H(z)=1-a(z)\left[\frac{A_{\min }^{-2}-A_{\max }^{-2}}{2}\right], \quad A_{0}(z)=1-a(z)\left[\frac{2 A_{\min }-1}{2 A_{\min }^{2}}-\frac{2 A_{\min }-1}{2 A_{\max }^{2}}\right] .
$$

Physically, this corresponds to modelling each cluster as an isothermal sphere in which we have truncated the lens profile at the angular size distance corresponding to an amplitude of $A_{\min }$, smoothed the distribution of matter within a core radius corresponding to $A_{\max }$, and imposed a net demagnification of objects relative to their amplitudes in a homogeneous universe.

In order to study the clustering properties of lensed objects, we examine a similar model for the two point amplification function

$$
\begin{aligned}
F\left(A_{1}, z_{1}, A_{2}, z_{2}, \theta\right)= & H\left(z_{1}, z_{2}, \theta\right) \delta\left(A_{1}-A_{0}\left(z_{1}, z_{2}, \theta\right)\right) \delta\left(A_{2}-A_{0}\left(z_{1}, z_{2}, \theta\right)\right) \\
+ & A_{1}^{-3} A_{2}^{-3} \theta\left(A_{1}-A_{\min }\right) \theta\left(A_{2}-A_{\min }\right) \times \\
& \theta\left(A_{\max }-A_{1}\right) \theta\left(A_{\max }-A_{2}\right) a\left(z_{1}\right) a\left(z_{2}\right)[1+w(\theta)]
\end{aligned}
$$


where $H$ and $A_{0}$ are fixed by conservation of probability at all values of redshift and angular separation. Imposing these conditions gives us

$$
\begin{aligned}
& H(z 1, z 2, \theta)=1-a\left(z_{1}\right) a\left(z_{2}\right)[1+w(\theta)]\left[\frac{1}{2 A_{\min }^{2}}-\frac{1}{2 A_{\max }^{2}}\right]^{2} \\
& A_{0}(z 1, z 2, \theta)=1-a\left(z_{1}\right) a\left(z_{2}\right) \frac{1+w(\theta)}{2}\left[\left(\frac{1}{A_{\min }}-\frac{1}{A_{\max }}\right)^{2}-\left(\frac{1}{2 A_{\min }^{2}}-\frac{1}{2 A_{\max }^{2}}\right)^{2}\right]
\end{aligned}
$$

Fixing the typical unlensed size of a submillimeter source to be $25 h^{-1} \mathrm{kpc}$ and the redshifts of a typical cluster and submillimeter source to be 0.5 and 3 respectively, gives a value of $A_{\max }=70$ in a flat universe $\left(\Omega_{\mathrm{M}}=1\right)$, with higher values for open cases. Throughout this work we fix $A_{\max }=50$, as a conservative limit. Somewhat more arbitrarily, we have set a minimum threshold of $A_{\min }=2$, because it is a round number above which more sophisticated lensing models typically display $F(A, z) \propto A^{-3}$ profiles (Peacock 1982; Pei 1995). We have examined the effect of these cutoffs on our model of cluster lensing and find that changing them introduces less than $20 \%$ uncertainty in our results for reasonable value of $A_{\max }$ and $A_{\min }$ and relevant threshold fluxes.

\section{Submillimeter Sources}

\subsection{A Semi-Empirical Model}

We use the simple model of TSB for the evolution of sources in the infra-red and submillimeter. The model evolves the properties of the local infra-red and submillimeter sources backwards in time, accounting for dust, gas, and spectral evolution. It agrees well with observations and constraints of the extra-galactic background from $\sim 5$ to $1000 \mu \mathrm{m}$ (Puget et al. 1996; Fixsen et al. 1998; Hauser et al. 1998; Biller et al. 1998), as well as with number count surveys from ISO (Puget et al. 1999; Aussel et al. 1998; Altieri et al. 1998) and SCUBA (Smail et al. 1998). Two types of sources, disk galaxies and starbursts, have significant fluxes in the submillimeter regime due to the reprocessing of stellar radiation by dust.

Disk submillimeter luminosity evolution is derived from observations of the star formation rate and metallicity histories of the Milky Way. A Schmidt Law relation (Kennicutt 1998) between star formation rate and gas density is used to derive an optical depth history. The contributions from both young and old stars to dust heating are modelled. The number evolution of disks is obtained from models of collision-induced 
galaxy formation (Balland, Silk, \& Schaeffer 1998) which attributes the formation of different morphological types to galaxy-galaxy interactions.

We model starburst evolution assuming they result from the mergers of gas-rich disks. The typical luminosity increases with the gas fraction, but decreases with the mean mass of the galaxies. The starburst number density is proportional to the instantaneous galaxy-galaxy collision rate.

These evolutionary models are applied to the present day far infra-red luminosity function, as observed by IRAS from 40 to $120 \mu \mathrm{m}$ (Saunders et al. 1990), assuming an Einstein-de Sitter cosmology with $h=0.5$. Given the uncertainties in the lensing model due to the evolution of clusters, we have not extended the submillimeter model to other cosmologies, but rather we restrict our attention to this cosmology. As lensing is more severe in open models and models with nonzero $\Omega_{\Lambda}$, these are expected to yield results above the $\alpha=2.9, \Omega_{\mathrm{M}}=1$ model, although we still consider the $\alpha=0.7, \Omega_{\mathrm{M}}=1$ model we examine below to be an overestimate of lensing even compared to $\Omega_{\mathrm{M}}<1$ cosmologies.

For simplicity, a cut is made in luminosity at $L_{\text {cut }} \sim 6 \times 10^{10} h^{-2} L_{\odot}$, below which sources are treated as disks, and above which they are treated as starbursts (Sanders \& Mirabel 1996). The results show the most sensitivity to this parameter, and to illustrate model uncertainties, we investigate the effect of varying $L_{\text {cut }}$ in Sections 3.2 and 4.

The luminosity function is also divided empirically into different spectral classes (Malkan \& Stecker 1998), and these spectra are analytically extended beyond $400 \mu \mathrm{m}$ into the submillimeter regime, assuming a dust emissivity dependence of $\nu^{\beta}$. Here we fix $\beta=1.5$ (Franceschini, Andreani, \& Danese 1998; Roche \& Chandler 1993), but our results are quite insensitive to this value as most of the sources detected are at $z \gtrsim 1$.

\subsection{Lensed Submillimeter Sources}

Applying Eq. 10 and 11 to our model for number counts of submillimeter objects as a function of flux and redshift, we are able to calculate the perturbation to the number of sources per unit flux due to lensing by clusters. If we take $\frac{d N_{\text {total }}}{d S}=\frac{d N_{\text {unlensed }}}{d S}+\Delta \frac{d N}{d S}$ then

$$
\begin{aligned}
\Delta \frac{d N}{d S}= & {\left[\frac{2 A_{\min }-2}{2 A_{\min }^{2}}-\frac{2 A_{\max }-2}{2 A_{\max }^{2}}\right] \frac{d N_{L}}{d S} } \\
+ & {\left[\frac{2 A_{\min }-1}{2 A_{\min }^{2}}-\frac{2 A_{\max }-1}{2 A_{\max }^{2}}\right] S \frac{d^{2} N_{L}}{d^{2} S}+\int_{A_{\min }}^{A_{\max }} \frac{d A}{A^{4}} \frac{d N_{L}}{d S}(S / A) }
\end{aligned}
$$


where $\frac{d N_{L}}{d S} \equiv \int_{0}^{\infty} a(z) \frac{d N}{d S d z} d z$. Notice that both $\int_{0}^{\infty} d S \Delta \frac{d N}{d S}$ and $\int_{0}^{\infty} S d S \Delta \frac{d N}{d S}$ are zero, as the lensing of objects does not change the total number of observed objects or the total integrated flux from those objects. Suppose, however, we consider only objects above a certain threshold flux $S_{\text {cut }} \cdot \int_{S_{\text {cut }}}^{\infty} d S \Delta \frac{d N}{d S}$ and $\int_{S_{\text {cut }}}^{\infty} S d S \Delta \frac{d N}{d S}$ need not be zero, as both the total number of objects above a given threshold and the total contribution to the flux by these objects can vary according to the degree of lensing. Furthermore, the total change above a threshold must be equal to the change below that threshold. Thus

$$
\int_{S_{\mathrm{cut}}}^{\infty} d S \Delta \frac{d N}{d S}=-\int_{0}^{S_{\mathrm{cut}}} d S \Delta \frac{d N}{d S}, \quad \text { and } \quad \int_{S_{\mathrm{cut}}}^{\infty} S d S \Delta \frac{d N}{d S}=-\int_{0}^{S_{\mathrm{cut}}} S d S \Delta \frac{d N}{d S},
$$

for all values of $S_{\text {cut }}$, and we are free to consider these quantities interchangeably.

By applying Eq. 12 and 13, the perturbation to $\frac{d^{2} N^{2}}{d S_{1} d S_{2}}$ due to lensing can be similarly calculated. Here we find

$$
\begin{aligned}
\Delta \frac{d^{2} N^{2}}{d S_{1} d S_{2}}= & (1+w(\theta))\left\{\left[\left(\frac{1}{A_{\min }}-\frac{1}{A_{\max }}\right)^{2}-2\left(\frac{1}{2 A_{\min }^{2}}-\frac{1}{2 A_{\max }^{2}}\right)^{2}\right] \frac{d N_{L}}{d S_{1}} \frac{d N_{L}}{d S_{2}}\right. \\
& +\frac{1}{2}\left[\left(\frac{1}{A_{\min }}-\frac{1}{A_{\max }}\right)^{2}-\left(\frac{1}{2 A_{\min }^{2}}-\frac{1}{2 A_{\max }^{2}}\right)^{2}\right]\left(S_{1} \frac{d^{2} N_{L}}{d S_{1}^{2}} \frac{d N_{L}}{d S_{2}}+\frac{d N_{L}}{d S_{1}} S_{2} \frac{d^{2} N_{L}}{d S_{2}^{2}}\right) \\
& \left.+\left(\int_{A_{\min }}^{A_{\max }} \frac{d A_{1}}{A_{1}^{4}} \frac{d N_{L}}{d S_{1}}\left(S_{1} / A\right)\right)\left(\int_{A_{\min }}^{A_{\max }} \frac{d A_{2}}{A_{2}^{4}} \frac{d N_{L}}{d S_{2}}\left(S_{2} / A\right)\right)\right\} .
\end{aligned}
$$

We now restrict our attention to flat cosmologies with $h=0.5$. Given the uncertainties in our knowledge of clusters and submillimeter sources, we expect this model to be sufficiently representative to examine the importance of structure induced by lensing. Due to the large volume and degree of lensing in open and cosmological constant models, we expect $\alpha=2.9, \Omega_{\mathrm{M}}<1$ models to fall somewhere between our Einstein-de Sitter $\alpha=2.9$ and slowly evolving cluster models.

In Fig. 3 we plot the total number of objects above a given threshold flux as a function of $S_{\text {cut }}$ as well as the change due to lensing by clusters at $850 \mu \mathrm{m}$, roughly corresponding to the SCUBA observations and one of the high-frequency channels on the Planck satellite. The model shown in this figure is not fine tuned to match the sub-mm observations, but rather is one example of a range of possible models. While the number of strongly lensed objects is equal to $\Delta N\left(>S_{\text {cut }}\right)$ for large values $S_{\text {cut }}$, deamplification causes the net perturbation to $N\left(>S_{\text {cut }}\right)$ to be an order of magnitude smaller than the number of strongly lensed objects at lower $S_{\text {cut }}$ values.

As noted by Peacock (1982), gravitational lensing is most important when $\frac{d N}{d S}$ is steeper than $S^{-3}$ and is able to overcome the $A^{-3}$ dependence of the high-magnification tail of 


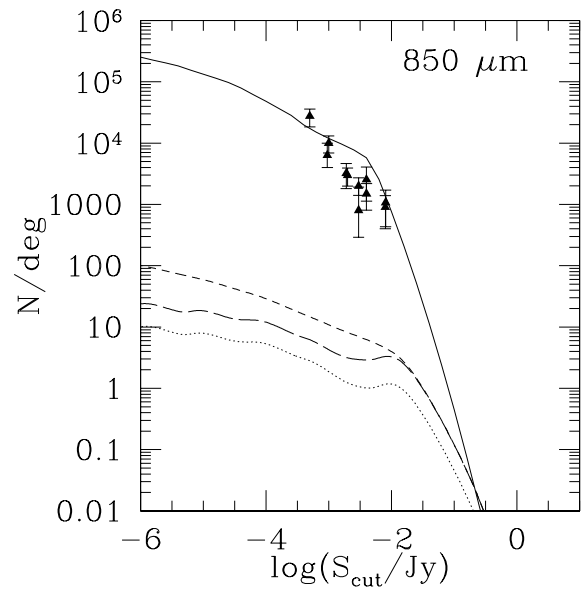

Fig. 3.- Number density of resolved objects as function of flux threshold at $850 \mu \mathrm{m}$. The solid line is the total number density of objects, $N\left(>S_{\text {cut }}\right)$, from TSB. Note that his particular model is not fine tuned to match the sub-mm observations, but rather is one example of a range of possible models. The lower curves show the perturbation due to lensing. For the $\Omega_{\mathrm{M}}=1, \alpha=0.7$ model the short-dashed line shows the change in number densities due to strong lensing by clusters, $\Delta N\left(>S_{\text {cut }}\right)$, and the long-dashed line is the number of strongly lensed objects (the contribution due to the last term of Eq. 14). The dotted curve shows $\Delta N\left(>S_{\text {cut }}\right)$ for the FBC Einstein-de Sitter model with $\alpha=2.9$. The points are the SCUBA data as tabulated by Smail et al. (1998). All errorbars are $1 \sigma$. 
the lensing distribution. For more shallow distributions, however, the degree of lensing is smaller by several orders of magnitude, and can even result in a net decrement to $N\left(>S_{\text {cut }}\right)$ for sufficiently shallow slopes (Broadhurst, Taylor, \& Peacock 1995). Thus the difference between the number of strongly lensed objects and $\Delta N\left(>S_{\text {cut }}\right)$ becomes important at values roughly corresponding to the turnover in the number counts of submillimeter objects. In the $\alpha=2.9$ and slowly evolving models $\Delta N\left(>S_{\text {cut }}\right)$ is almost of the same form, but is smaller for the slowly evolving model by a factor $\sim 3$.

In Fig. 1 we compare flux per unit angle due to unresolved objects in lensed and unlensed distributions as a function of flux cut at $850 \mu \mathrm{m}$. As objects behind gravitational lenses are fewer and more luminous, imposing an overall flux cut causes a stronger decrement in the lensed distribution of objects than in the unlensed case. Again, this flux decrement in a slowly evolving model is of the same form as in the FBC model, but scaled down by a factor $\sim 3$. Note that while the flux of lensed objects is only $\sim 0.1 \%$ of the total flux, the correlations induced by clustering can make this contribution important at large angular scales.

This discrepancy in fluxes also raises the question of the clustering of the objects themselves. While this is likely to be weak and at very small angles, Scott \& White (1998) have demonstrated that this clustering may be dominant over Poisson noise at angles $\sim 2^{\prime}$, although their model is only for illustrative purposes. As this contribution goes down as a function of flux-cut, and is important only at small angles, for our purposes it is best thought of as an uncertainty in the small-scale noise and will turn out not to affect our results.

Our models are quite sensitive to the threshold between starburst and disk galaxies. Raising the value of $L_{\text {cut }}$ suppresses the high- $z$ luminous sources, shifting the turnover point in the $\frac{d N}{d S}$ curve. As the lensed flux is extremely sensitive to this slope, the number counts and flux decrements in these model differ dramatically. In Fig. 5 we consider two submillimeter models that overpredict and underpredict this slope. The steep $\left(L_{\text {cut }}=3 \times 10^{10} h^{-2} L_{\odot}\right)$ model displays a large peak in the number counts and flux decrements that is absent in the more shallow $\left(L_{\text {cut }}=12 \times 10^{10} h^{-2} L_{\odot}\right)$ model. While these are extreme models, chosen to make a point, ignorance of this slope is nevertheless one of the largest uncertainties in our analysis. 


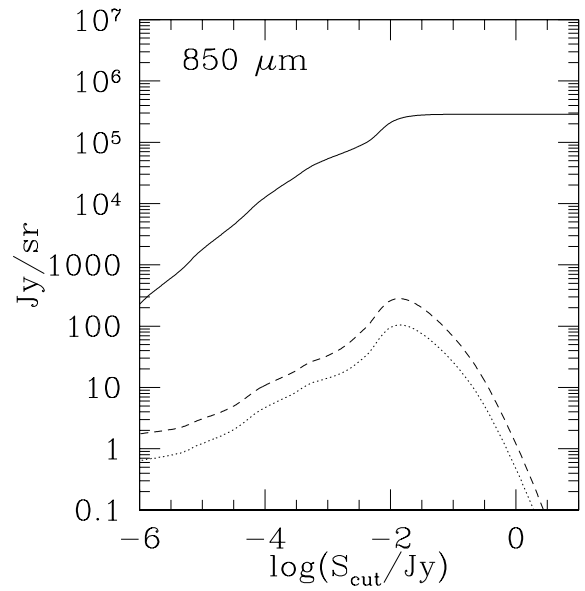

Fig. 4.- Flux per unit steradian in unresolved objects as a function of flux threshold at 850 $\mu \mathrm{m}$. The solid line is unlensed flux, the dashed line is the decrement in flux due to lensing by clusters in the slowly evolving model $\left(\Omega_{\mathrm{M}}=1, \alpha=0.7\right)$ and the dotted line is the flux decrement in the $\Omega_{\mathrm{M}}=1, \alpha=2.9$, model.

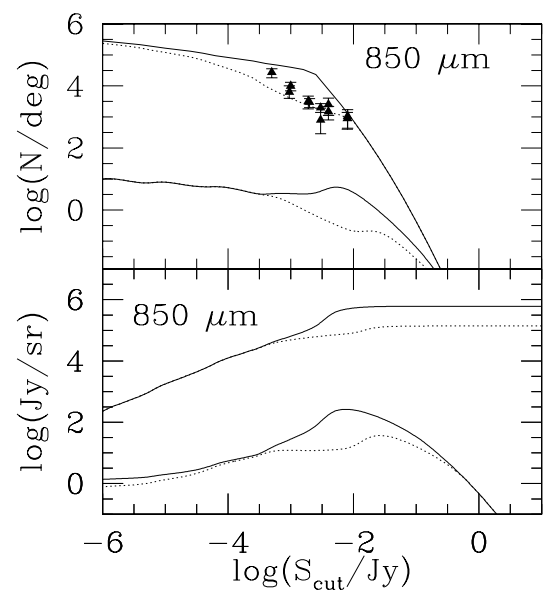

Fig. 5.- The effect of uncertainties in the submillimeter model on lensing. In the upper panel, the upper curves show the total number of objects without lensing by clusters, $N\left(>S_{\text {cut }}\right)$, for two different models while the lower curves show the change in this number density due to lensing, $\Delta N\left(>S_{\text {cut }}\right)$. Here $L_{\text {cut }}=3 \times 10^{10} h^{-2} L_{\odot}$ is represented by the solid lines, and the dotted lines represent $L_{\text {cut }}=12 \times 10^{10} h^{-2} L_{\odot}$. In the lower panel, the upper curves show total unlensed fluxes and the lower curves show the flux decrement due to lensing, with the lines corresponding to models as in the upper panel. 


\section{Background Anisotropies}

Contributions of point sources to background anisotropies can arise in two different ways: Poisson noise due to the intrinsic graininess of the distribution, and structured noise due to the placement of the sources on the sky. The first source of noise is given by

$$
C_{\ell}^{I}=\int_{0}^{S_{\mathrm{cut}}} d S S^{2} \frac{d N_{\mathrm{Tot}}}{d S}=\int_{0}^{S_{\mathrm{cut}}} d S S^{2}\left(\frac{d N}{d S}+\Delta \frac{d N}{d S}\right)
$$

where we use the notation $C_{\ell}^{I}$ to denote the angular power spectrum in units of intensity rather than the usual decomposition in terms of the temperature fluctuations. $C_{\ell}^{I}$ is related to the usual $C_{\ell}$ by

$$
C_{\ell}=C_{\ell}^{I}\left(T_{\mathrm{CMB}} \frac{\partial B_{\nu}}{\partial T}\right)^{-2}=C_{\ell}^{I}\left(2.71 \times 10^{10} \mathrm{mJy} \mathrm{sr}^{-1} \frac{x^{4} e^{x}}{\left(e^{x}-1\right)^{2}}\right)^{-2}
$$

where $x \equiv h \nu / k_{B} T_{\mathrm{CMB}}=\nu / 56.84 \mathrm{GHz}$ and $1 \mathrm{Jy}=10^{-26} \mathrm{Wm}^{-2} \mathrm{~Hz}^{-1}$ (Scott \& White 1998). Note that here we follow the conventional notation for microwave background fluctuations, expressing our results in terms of $C_{\ell} \mathrm{s}$, given by the Legendre transform of the correlation function $C(\theta)$.

As lensing skews the distribution by gathering the flux into fewer, brighter objects, it does slightly increase the Poisson noise of the observations. This contribution to the anisotropies is a single-point process, however, with no intrinsic scale, and is completely independent of the placement of the lensing objects on the sky. The level of Poisson noise in our model and the increment due to lensing are shown in Fig. 6. Notice that removing point sources above a certain flux only decreases the Poisson noise, as it reduces the total power in the grainy distribution.

The second contribution of point sources to anisotropies, that of inhomogeneities in the mean flux as a function of position is given by

$$
C(\theta)^{I}=\int_{0}^{S_{\mathrm{cut}}} S_{1} d S_{1} \int_{0}^{S_{\mathrm{cut}}} S_{2} d S_{2}\left[\Delta \frac{d N^{2}\left(S_{1}, S_{2}, \theta\right)}{d S_{1} d S_{2}}\right]
$$

where $\Delta \frac{d N\left(S_{1}, S_{2}, \theta\right)}{d S_{1} d S_{2}}$ is as given in Eq. 16. Note that at this point we are ignoring intrinsic correlations of the submillimeter objects themselves. While this is a concern at high $\ell$ values $\gtrsim 500$ corresponding to the galaxy-galaxy correlation scale (Scott and White 1998), it introduces little uncertainty into the analysis at $\ell$ s corresponding to cluster correlations.

If one imposes no upper limit on the flux of the point sources, then the lensing cannot add structure to the angular correlations of point sources. This is due to the conservation 
of total flux behind the lenses, Eq. 15, which insures that statistically, the average flux from a lensed pixel will be equal to that of unlensed areas of the sky. Unlike Poisson noise, imposing a finite flux cut by removing point sources actually increases this contribution to anisotropies as it skews the mean flux from lensed pixels to lower values. This introduces a flux anisotropy where there previously was none, adding power on the angular scale given by the clustering of the lenses.

We can calculate the magnitude of this effect from the Legendre transform of Eq. 19.

$$
\begin{aligned}
C_{\ell}^{I} & =w_{\ell}\left\{\left.\left[\left(\frac{1}{A_{\min }}-\frac{1}{A_{\max }}\right)^{2}-\left(\frac{1}{2 A_{\min }^{2}}-\frac{1}{2 A_{\max }^{2}}\right)^{2}\right] S_{\mathrm{cut}}^{2} \frac{d N_{L}}{d S_{1}}\right|_{S=S_{\mathrm{cut}}} \int_{0}^{S_{\mathrm{cut}}} d S_{2} S_{2} \frac{d N_{L}}{d S_{2}}\right. \\
& -\left(\frac{1}{A_{\min }^{2}}-\frac{1}{A_{\max }^{2}}\right) \int_{0}^{S_{\mathrm{cut}}} d S_{1} S_{1} \frac{d N_{L}}{d S_{1}} \int_{0}^{S_{\mathrm{cut}}} d S_{2} S_{2} \frac{d N_{L}}{d S_{2}} \\
& \left.+\left(\int_{0}^{S_{\mathrm{cut}}} d S_{1} S_{1} \int_{A_{\min }}^{A_{\max }} \frac{d A_{1}}{A_{1}^{4}} \frac{d N_{L}}{d S_{1}}\left(S_{1} / A\right)\right)\left(\int_{0}^{S_{\mathrm{cut}}} d S_{2} S_{2} \int_{A_{\min }}^{A_{\max }} \frac{d A_{2}}{A_{2}^{4}} \frac{d N_{L}}{d S_{2}}\left(S_{2} / A\right)\right)\right\},(20)
\end{aligned}
$$

where $w_{\ell} \approx 2 \pi \theta_{0}^{-\gamma-1}\left(\ell^{-\gamma-3}\right)$. This expression is only at $\ell$ values below the angular size of a cluster at a typical redshift $\sim 0.5$, and at $\ell$ values above which a power law fit to the angular correlation function of galaxies is appropriate $\left(5^{\circ} \gtrsim \theta \gtrsim 1^{\prime}\right.$ or $\left.10 \lesssim \ell \lesssim 1000\right)$ (Tadros, Efstathiou, \& Dalton 1998).

In Fig. 7 we plot this contribution to the anisotropy of unresolved sources along with the Poisson noise anisotropy at $\ell=1 /\left(1.1^{\circ}\right)=27$. As $C_{\ell}$ is proportional to $\left(\frac{d N_{L}}{d S}\right)^{2}$, the slowly evolving lensing model gives a contribution $\sim 25$ greater than the $\alpha=2.9$ model. Note also that this comparison is only valid at this particular $\ell$ value as the Poisson noise and lensing anisotropy have different scalings as a function of $\ell$, with $\ell^{2} C_{\ell}$ of the lensing contribution going as $\ell^{-\gamma-1}$, and $\ell^{2} C_{\ell}$ of Poisson noise going as $\ell^{2}$. Thus, at higher $\ell$ values, Poisson noise will be more dominant and will be even more enhanced at very high $\ell$ values by galaxy-galaxy correlations, while at lower values the lensing anisotropy will play a larger role. For reference, the expected microwave background contribution is given by $\ell^{2} C_{\ell} / 2 \pi \approx 10^{-10}$ for $\ell \lesssim 1000$.

In Fig. 8 we plot the same quantities, but now for the $L_{\text {cut }}=3 \times 10^{10} h^{-2} L_{\odot}$ submillimeter model. While this model slightly overproduces the background flux and most likely overpredicts both lensing and Poisson anisotropies, it nevertheless serves to illustrate the sensitivity of our results to the slope of the number counts near the flux-cut threshold. While Poisson noise is boosted only by a factor of two in this model, the lensing anisotropy is increased by more than an order of magnitude by a change in this slope. Nevertheless, even in this extreme model, the FBC lensing anisotropy falls short of the Poisson noise.

Finally, in Fig. 9 we compare Poisson noise and noise induced by lensing at $550 \mu \mathrm{m}$ 


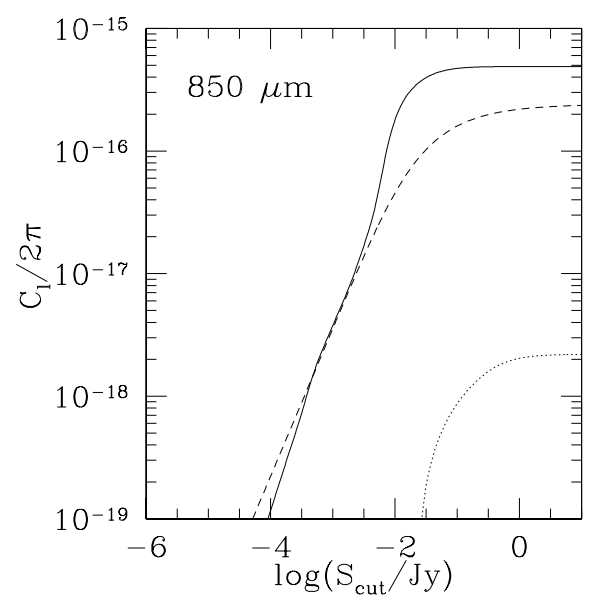

Fig. 6.- Contribution of confusion noise to measurements of $C_{\ell} / 2 \pi$ as a function of flux cut The solid line is unlensed flux while the dotted line is the increment in this flux due to lensing by clusters. The dashed line is the Poisson noise as calculated from a simple double-power law fit by Scott \& White (1998).

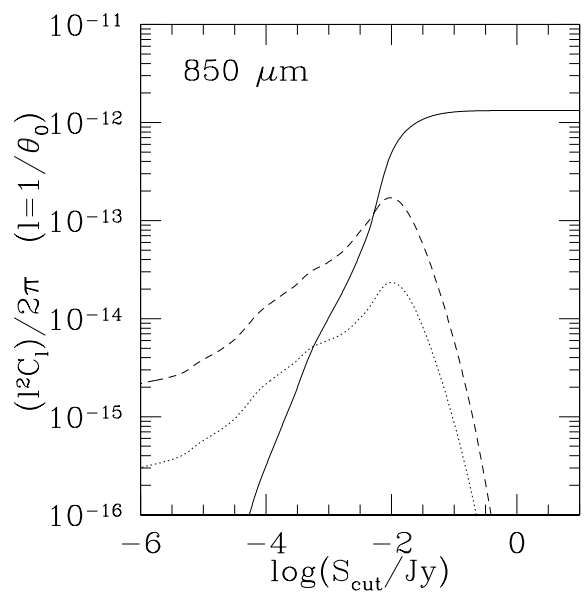

Fig. 7.- Contribution of lensing-induced structure to measurements of $\ell^{2} C_{\ell} / 2 \pi$ as a function of flux cut at $\ell=1 / \theta_{0}$, the correlation angle. The solid line is the Poisson noise of the unlensed distribution assuming $\theta_{0}=1.1^{\circ}$, the dashed line is the anisotropy due to lensing in the slowly evolving cluster model, and the dotted line is the anisotropy in the FBC evolution model. 


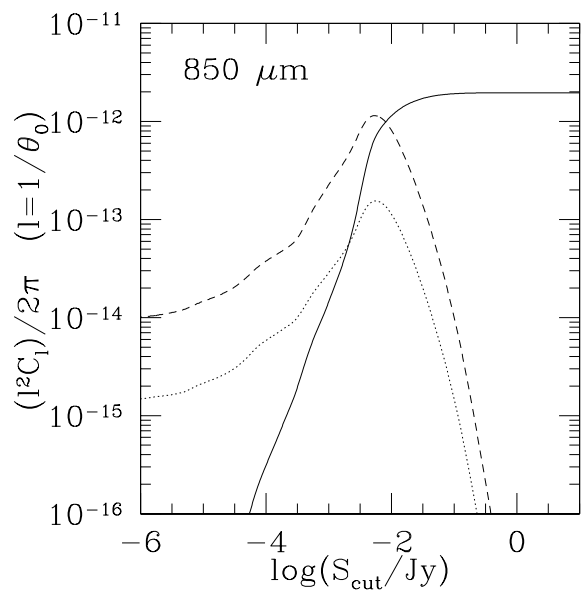

Fig. 8. - Contribution of lensing-induced structure to measurements of $\ell^{2} C_{\ell} / 2 \pi$ at $\ell=1 / \theta_{0}$. as a function of flux cut, for a model $\left(L\right.$ cut $\left.=3 \times 10^{10} h^{-2} L_{\odot}\right)$ with a steeper source count slope near the flux cut The curves are as in Fig. 7 .

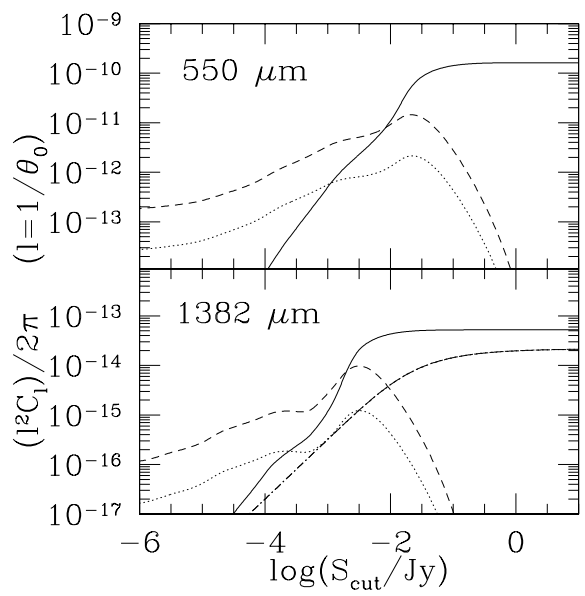

Fig. 9.- Contribution of lensing-induced structure to measurements of $\ell^{2} C_{\ell} / 2 \pi$ at $\ell=1 / \theta_{0}$ as a function of flux cut at two different wavelengths. The upper panel corresponds to the $550 \mu \mathrm{m}, 545 \mathrm{GHz}$ Planck channel, with the curves as in Fig. 7. The lower panel is the same comparison for the $1382 \mu \mathrm{m}, 216 \mathrm{GHz}$ Planck channel. The solid, short-dashed, and dotted curves are as before, while the additional long-dashed curve is the simple extrapolation by Scott and White (1998). 
and $1382 \mu \mathrm{m}$, which correspond to the Planck high-frequency channels above and below the $850 \mu \mathrm{m}$ channel we have considered so far. Again our results indicate that Poisson noise is dominant over structure induced by lensing for realistic models of clustering. Note, however, that the level of submillimeter anisotropies relative to microwave background anisotropies are drastically effected by changes in wavelength. At $545 \mathrm{GHz}$ we expect background measurements to be dominated by point source contributions, that is, the far-infrared background. At $217 \mathrm{GHz}$, however, point source contributions are much reduced, becoming comparable to microwave anisotropies only at scales smaller than $\ell \sim 20 / \theta_{0}=1000$. As a side note we point out that our semi-empirical model shows a much sharper decrease in Poisson noise in the 1 to $10 \mathrm{mJy}$ range at $217 \mathrm{GHz}$ than the simple extrapolation by Scott \& White (1998), due to the sharper turnover of $\frac{d N}{d S}$ in the TSB model. Thus high- $\ell$ microwave anisotropy measurements at this frequency may gain more from a careful subtraction of point sources than previously expected.

\section{Conclusions}

While the surface density of submillimeter objects was previously unknown to within a factor of a thousand, recent observations have given us our first glimpse into the submillimeter universe. This breakthrough has allowed authors to quantify the level of background emission, confusion limits on future observations, and the effects of lensing on the number counts of resolved objects.

In this work, we have examined the effect of lensing by clusters on the angular distribution of submillimeter sources. While lensing does not affect the total flux, it can cause a flux decrement of unresolved lensed sources in flux-limited samples. In the case of lensing by clusters, this flux decrement is correlated on scales corresponding to the cluster correlation length $\sim 1.1^{\circ}$, and thus represents a possible contribution to background anisotropies at microwave and far-infrared wavelengths.

Using a simple model for lensing by clusters and a semi-empirical model for the evolution of lensed objects, we have quantified this contribution, relating it to the anisotropy due to the discreteness of the unresolved source distribution. While slowly evolving cluster models predict structure comparable to Poisson noise on angles $\sim 1.1^{\circ}$, more likely cluster models predict anisotropies to be dominated by Poisson noise. The degree of structure induced in an $\Omega_{\mathrm{M}}<1$ universe is expected to be more than for the "best guess", $\Omega_{\mathrm{M}}=1$ model, but still fall short of the slowly evolving case we have considered.

Throughout this work we have assumed a uniform distribution of submillimeter objects 
and have not addressed the question of the clustering of the sources themselves. This clustering length is much shorter than that of galaxy clusters and the clustering weaker, but it nevertheless may represent a significant contribution at small angles. At larger angles this effect is negligible, however, and thus the issue of structure in the far-infrared background itself does not effect the conclusions drawn here. It is with some confidence then that we can say that experiments measuring microwave background anisotropies and far-infrared background anisotropies due to the clustering of unresolved sources will be able to remove all resolved point sources without inducing structure in unresolved sources from lensing by galaxy clusters.

\section{Acknowledgements}

We appreciate valuable input from Rychard Bouwens, Tom Broadhurst, R. Benton Metcalf, D. M. Sherfesee, and Edward L. Wright.

\section{REFERENCES}

Abadi, M. G., Lambas, D. G., \& Muriel, H. 1998, ApJ, 507, 526

Altieri et al. 1998, A\&A, accepted, astro-ph/9810480

Aussel, H., Cesarsky, C. J., Elbaz, D., \& Starck, J. L. 1998, A\&A, 342, 313

Balland, C., Silk, J., \& Schaeffer, R. 1998, ApJ, 497, 541

Bahcall, N. A., Fan. X., \& Cen, R. 1997, ApJ, 485, L53

Bahcall, N. A., \& Cen R., 1992, ApJ, 398, L81

Bardeen J. M., Bond, J. R., Kaiser, N., Szalay, A. S. 1986, ApJ, 304, 15

Barger, A. J. 1998, Nature, 394, 248

Bersanelli, M. et al. 1996, COBRAS/SAMBA, SCI(96)3, ESA, Paris

Biller, S. D. et al. 1998, PRL, 80, 2992

Blain, A. W. 1996, MNRAS 283, 1340

Blain, A. W. 1997, MNRAS 290, 553

Blain, A. W., 1998, MNRAS, 297, 511

Blain, A.W., Ivison, R. J., \& Smail, I. 1998, MNRAS, 296, L29

Blain, A.W., Ivison, R. J., Smail, I., \& Kneib J. P. 1998 to appear in "Wide-field surveys in cosmology", proc. XIV IAP meeting (astro-ph/9806063)

Blain, A. W., \& Longair, M. S. 1993, MNRAS, 264, 509

Blain, A. W., \& Longair, M. S. 1996, MNRAS, 279, 847 
Blanchard, A. \& Bartlett, J. G. 1998, 332, L49

Broadhurst, T., Taylor, A. N., \& Peacock, J.A. 1995, ApJ, 428, 49

Carlberg, R. G., Morris, S. L., Yee, H. K. C., \& Ellingson, E. 1997, ApJ, 479, L19

Carroll, S. M., \& Press, W. H. 1992, ARAA, 30, 499

Close, L. M., Hall, P. B., Liu, M. C., Hege, E. K., 1995, ApJ, 452, L9

Dalton, G. B., Maddox, S. J., Sutherland, W. J., \& Efstathiou, G. 1994, MNRAS, 271, L47

Dyer, C. C., \& Roeder R. C. 1973, ApJ, 180, L31

Eales et al. 1998, ApJ, submitted, (astro-ph/9808040)

Eke, V. R., Cole, S., \& Frenck, C. S. 1996, MNRAS, 282, 263

Fan, X., Bahcall, N. A., \& Cen, R. 1997, ApJ, 490, L123 (FBC)

Fixsen, D.J., Dwek, E., Mather, J.C., Bennett, C.L., \& Shafer, R.A. 1998, ApJ, 508, 123

Franceschini, A., Toffolatti, H., Mazzei, P., Danese, L., \& de Zotti, G. 1991, A\&A, 89, 285

Franceschini, A., Andreani, P., \& Danese L. 1998, MNRAS, 296, 709

Hauser, M. G. et al. 1998, ApJ, 508, 25

Henry, J. P., \& Arnaud, K. A. 1991, ApJ, 372, 410

Henry, J. P., Gioia, I. M., Maccacaro, T., Morris, S. L., Stocke, J. T., \& Wolter, A. 1992, ApJ, 386, 408

Holland, W. S. et al. 1999, MNRAS, 303, 659

Hughes, D. et al. 1998, Nature, 394, 241

Kennicutt, R. C. 1998, ApJ, 498, 541

Malkan, M. A., \& Stecker, F.W. 1998, ApJ, 496, 13

Navarro, J. F., Frenck, C. S., \& White, S. D. M. 1996, ApJ, 462, 563

Nichol, R. C., Collins, C. A., Guzzo, L., \& Lumsden, S. L. 1992, MNRAS, 254, 692

Peacock, J. A. 1982, MNRAS, 199, 987

Peacock, J. A. 1986, MNRAS, 223, 113

Pei, Y. C. 1993a, ApJ, 403, 7

Pei, Y. C. 1993b, ApJ, 404, 436

Pei, Y. C. 1995, ApJ, 440, 485

Press, W. H., \& Schechter, P. 1974, ApJ, 187, 425

Puget, J. L. et al. 1996, A\&A, 308, L5

Puget, J. L. et al. 1999, A\&A, 345, 29

Roche, P. F., \& Chandler, C. J. 1993, MNRAS, 265, 486

Sadat, R., Blanchard, A., \& Oukbir, J., 1998, A\&A, 329, 21

Sanders, D. B., \& Mirabel, I. F. 1996, ARAA, 34, 749 
Saunders, W., Rowan-Robinson, M., Lawrence, A., Efstathiou, G., Kaiser, N, Ellis R. S., \& Frenk C. S. 1990, MNRAS, 242, 318

Schlegel, D., Finkbeiner, D., \& Davis, M. 1998, ApJ, 500, 525

Scott, D., \& White, M. 1998, A\&A, accepted, astro-ph/9808003)

Smail, I., Ivison, R. J., \& Blain, A. W. 1997, ApJ, 409, L5

Smail, I., Ivison, R. J., Blain, A. W., \& Kneib, J. P. 1998, Invited review at Maryland Astrophysics Conference "After the dark ages: when galaxies were young (the Universe at $2<\mathrm{z}<5$ )," (astro-ph/9810281)

Tadros, H., Efstathiou G., \& Dalton, G. 1998, MNRAS, 296, 995

Tan, J. C., Silk, J., \& Balland, C. 1999, ApJ, accepted, (astro-ph/9904004) (TSB)

Tyson, J. A., \& Fischer, P. 1995, ApJ, 466, L55

Vietri, M., \& Ostriker, J. P. 1982, ApJ, 267, 488

Weinberg, S. 1976, ApJ, 208, L1

Yee, H. K. C., Ellingson, E., \& Carlberg, R. G. 1996, ApJS, 102, 269

Young, P., Gunn, J., Kristian, J. Oke, J. B., \& Westphal, J. A. 1980, ApJ, 241, 507

Zabludoff, A. I., Geller, M. J., Huchra, J. P., \& Ramella, M. 1993, AJ, 106, 1301 